\title{
Efectos de las instrucciones audiovisuales no explícitas sobre el comportamiento alimentario*
}

\author{
Effects of non-explicit audiovisual instructions \\ on feeding behavior
}

\author{
Hortencia Alicia Barrera Pelayo \\ Antonio López-Espinoza** \\ Alma Gabriela Martínez \\ Carolina De La Torre-Ibarra \\ Virginia Aguilera \\ Alma Galindo \\ Felipe Díaz \\ Karina Franco \\ Claudia R. Magaña \\ Centro de Investigaciones \\ en Comportamiento \\ Alimentario y Nutrición (CICAN) \\ Cusur - Universidad de Guadalajara \\ Recibido: 2 de abril de 2009 \\ Revisado: 5 de junio de 2009 \\ Aceptado: 3 de agosto de 2009
}

\section{Resumen}

Este estudio evaluó los efectos de las instrucciones no explícitas sobre la conducta alimentaria. Participaron cinco jóvenes entre los 19 y 24 años, a quienes se les observó durante dos fases experimentales a dos tipos de alimento nutricional (fruta y agua) y no nutricional (papas fritas y refresco). Los participantes fueron filmados en dos programas de video. En la primera fase se grabaron videos musicales y en la segunda, un documental sobre hábitos alimentarios saludables. Los resultados mostraron que las instrucciones no explícitas modificaron la conducta alimentaria. Sin embargo, es necesario evaluar si estas modificaciones persisten a largo plazo.

Palabras clave: conducta alimentaria, consumo, instrucción, humanos.

\section{Abstract}

This study evaluated the effects of non-explicit audiovisual instructions on feeding behaviour. Five adults between 19 and 24 years-old participated as subjects, which were exposed at nutritional (fruit and water) or no nutritional (chips and soda) foods

Artículo de investigación. Esta investigación fue financiada por el proyecto PROMEP/103.5/05/1705 UDG-PTC-279 de la Universidad de Guadalajara. Correspondencia: doctor Antonio López-Espinoza. Centro de Investigaciones en Comportamiento Alimentario y Nutrición, Universidad de Guadalajara, Ciudad Guzmán, Municipio Zapotlán el Grande, Jalisco, México. Av. Prolongación Colón S/N Edificio X- 3. Km. 1 Carretera Ciudad Guzmán-Guadalajara, C.P. 49000. Correos electrónicos: Hortencia Alicia Barrera Pelayo: habpelayo@hotmail.com; Antonio López-Espinoza: antonio. lopez@cusur.udg.mx. 
during two experimental phases. Subjects were videotaped and exposed to two video programs in different phases. In the first phase, subjects were exposed to a musical video, in the second phase subjects were exposed to a program about health eating habits. The results showed that nonexplicit audiovisual instructions modified feeding behaviour. However, will be necessary evaluate if these modification could persist for longer time.

Key words: feeding behaviour, consumption, instruction, human.

\section{Introducción}

La evidencia científica ha demostrado que las instrucciones juegan un papel fundamental en el establecimiento del patrón alimenticio, la preferencia, la selección y su consumo (Young, 1966; Long, Montano, Conklin \& Scheer, 2003). Otros factores que influyen en la conducta alimentaria son aspectos propios del individuo, como el gusto o disgusto por determinada comida, el ambiente social (la familia, amigos) y el ambiente físico en que se desarrolla (la accesibilidad al alimento, los medios de comunicación) (Store, Neumark-Sztainer \& French, 2002).

Gran parte de la investigación sobre los efectos de las instrucciones en la conducta humana ha sido desarrollada por pedagogos y psicólogos conductuales. Szczurek (1978) y Gagne (1987) señalaron la importancia de la secuencia y del medio ambiente en el uso de instrucciones, a fin de contribuir en el proceso de aprendizaje. Subrayaron la distinción entre instrucción como producto e instrucción como proceso. En el primero, aludieron a un conocimiento claro y firme, y en el segundo, a la relación de dos conceptos: enseñanza y aprendizaje.

Diversos autores refieren la importancia de cómo se desarrolla la situación instruccional para la ejecución de una conducta (Szczurek, 1978; Gagne, 1987; Martínez, Ortiz \& González, 2002). Edmonds, Branco \& Mukherjee (1994) señalaron que son de importancia el tipo procedimental (cómo) $y$, el tipo declarativo (por qué). Señalaron que se debe tener en cuenta el nivel de competencia requerido, es decir, los conocimientos previos y las habilidades asociadas de quienes participaron en el proceso de instrucción, para que la instrucción sea lo más clara posible. Adicionalmente, el tipo de instrucción basado en el propósito puede ser descriptivo o prescriptivo, con ello se esquematiza cómo se puede manipular un ambiente a fin de llegar a la meta.

Martínez (2001) señaló que una misma instrucción puede ejercer control sobre ejecuciones distintas en diferentes sujetos, y que la ejecución de una misma instrucción puede variar de acuerdo con el momento en que sea emitida. Baron \& Galizio (1983) y, Martínez \& Ribes (1996) mencionaron la importancia de considerar como variables la historia individual, la instrucción específica y la historia del reforzamiento en el seguimiento instruccional a fin de obtener la conducta deseada al emitir una instrucción.

Por otra parte, el efecto de las instrucciones sobre la conducta alimentaria en humanos no es un tema nuevo, pero hay poca investigación al respecto. No obstante, va en aumento el interés por este tópico de profesionistas de diversas áreas, como: la nutrición, la medicina, la pedagogía, la psicología, entre otras.

Young (1966) señaló respecto a las instrucciones y la conducta alimentaria, que aceptar algún tipo de alimento es un proceso complejo que puede ser regulado por cuatro grupos de factores: a) las condiciones orgánicas; b) las estimulaciones periféricas como el gusto, el olfato, el tacto, la visión, y la audición, así como las condiciones ambientales en las que se encuentra la comida, su disponibilidad y consistencia; c) las experiencias previas (hábitos, actitudes); y, d) la constitución corporal (p.ej.: las diferencias entre individuos y especies).

Respecto a las condiciones orgánicas y a los estímulos periféricos en la conducta alimentaria Bellisle, Guy-Grand \& Le Magnen (2000) desa- 
rrollaron un experimento sobre la masticación y el tragar como índices del estímulo para la conducta de comer en seres humanos. Encontraron que cuando el sabor de la comida era agradable aumentaba la frecuencia y velocidad en la masticación y que al inicio de la exposición a la comida la cantidad de ingesta era mayor. Señalaron que la textura era un factor crítico para la ingesta de alimento. Por otra parte, Long et al., Montano, Conklin y Scheer (2003) analizaron la influencia de un programa educacional en un grupo de adultos y concluyeron que las instrucciones de orientación nutricional modificaron la forma en cómo piensan los participantes cambiar sus conductas de consumo de frutas y verduras.

Adicionalmente, se ha señalado que los medios de comunicación funcionan como instrucciones no explícitas promoviendo conductas alimenticias (Taras, Sallis, Patterson, Nader \& Nelson, 1989; Silverio \& Alves, 2004). Ejemplo de ello son los anuncios de golosinas, alimentos enlatados, productos dietéticos, entre otros. Esta particularidad ha motivado el desarrollo de campañas publicitarias con la finalidad de promover un patrón alimenticio saludable al utilizar estímulos audiovisuales como medio instruccional. Un ejemplo de este tipo de campaña es la denominada "Cinco por día" (OMS y FAO, 2005). Partiendo de la evidencia anterior, el objetivo general del presente estudio fue evaluar el papel de las instrucciones audiovisuales no explícitas sobre la conducta de consumo de alimento en humanos.

\section{Método}

\section{Sujetos}

Participaron cinco voluntarios (dos hombres y tres mujeres) entre los 19 y 24 años, experimentalmente ingenuos. Estudiantes de nivel licenciatura y profesionales, solteros, sin hijos y consumidores al menos en una ocasión de refresco de cola.

\section{Aparatos y materiales}

Se utilizó el laboratorio de conducta alimentaria del Centro de Investigación en Comportamiento
Alimentario y Nutrición (CICAN) que cuenta con cámara Gesell, acondicionado con tres video-cámaras discretas ubicadas en tres puntos. Se utilizaron cinco sillas y dos mesas, en una de ellas se colocó la bebida y los alimentos, y en la otra un televisor con un reproductor DVD. Se utilizaron dos programas pregrabados, el primero con videos musicales y el segundo con información sobre los efectos negativos del consumo excesivo de papas fritas y el refresco de cola. También se utilizaron dos bandejas para presentar los siguientes alimentos: $1 / 2 \mathrm{~kg}$ de jícama (nabo), $1 / 2 \mathrm{~kg}$ de pepino, $1 / 2 \mathrm{~kg}$ de mango y $1 / 2 \mathrm{~kg}$ de naranja. Como bebidas utilizamos 10 botellas de agua de $500 \mathrm{ml}$, 10 botellas de refresco de cola de $500 \mathrm{ml}$ ( 5 light y 5 normales). Cuatro paquetes de $500 \mathrm{mg}$ de papas fritas. Un paquete de 150 servilletas, tenedores, 50 platos desechables, hielo y tenazas.

Al finalizar el procedimiento experimental se aplicó un cuestionario para solicitar datos personales (nombre, edad, grado académico, estado civil); horarios (a qué hora despiertas, a qué hora desayunas, a qué hora prefieres determinado alimento); preferencia de alimento y bebida (qué sueles desayunar, qué prefieres para desayunar, fruta que más te gusta, fruta que menos te gusta); gusto o disgusto por los alimentos y bebidas propuestos en el experimento (¿algún alimento o bebida de las presentadas en el experimento no fue de tu agrado?); y, finalmente, se preguntó si habían desayunado antes de asistir al experimento (¿desayunaste antes de asistir aquí?, ¿a qué hora?, ¿qué desayunaste?).

\section{Procedimiento}

Se citaron a los jóvenes a una hora y fecha determinadas y se les dio la siguiente instrucción:

Se llevará a cabo un estudio de gran relevancia, en el cual se pretende conocer las diferentes habilidades que presentan hombres $y$ mujeres universitarios en la búsqueda de información en la red para la elaboración de sus trabajos académicos. Se les proporcionarán técnicas para una búsqueda ágil y eficaz. Dicho estudio se realizará en un aula del CEIC, 
que está equipada y acondicionada con el material que ustedes requieren: un escritorio con una pantalla y un teclado de computadora y una silla.

Previo al experimento se preparó el alimento y el material requerido. Dos horas antes del inicio del experimento se colocaron en hielera las botellas de agua y refresco. Luego se picó la fruta y se colocó en las charolas al igual que las papas fritas.

Fase 1. Una hora antes de iniciar el experimento, se colocaron cinco sillas y una mesa ubicada en el centro del cuarto experimental. Otra mesa fue ubicada en un extremo para poner el televisor y el reproductor de DVD.

Cuando llegaron los jóvenes se les solicitó que esperaran en el pasillo anexo al cuarto de experimentación. Posteriormente, fueron invitados a pasar al cuarto de experimentación bajo la indicación: "Por favor esperen en este cuarto mientras ajustamos los últimos detalles para el experimento en el que participarán". Inmediatamente comenzó la transmisión del DVD. Diez minutos después ingresaron dos investigadores para colocar las charolas con el alimento antes mencionado, las botellas de agua, de refresco y vasos desechables. Uno de los investigadores dio la siguiente indicación: "Tienen a su disposición una mesa con aperitivos. Debido a que este es un centro de investigación y se están haciendo otras investigaciones, no podrán salir del cuarto hasta que se les indique". El segundo investigador estuvo detrás de la cámara Gesell revisando el funcionamiento del equipo electrónico y registrando el comportamiento de los sujetos en estudio. Este investigador permaneció afuera del cuarto experimental contabilizando el tiempo y asesorando a los sujetos cuando fue necesario. Cuarenta minutos después se informó que: "Debido a fallas técnicas, el experimento se pospondrá al sábado próximo, a la misma hora y en el mismo lugar, esperando su puntual asistencia. Se agradeció de antemano la comprensión por no haber podido realizarlo en el tiempo y la forma que estaba previsto".
Fase 2. La indicación correspondiente fue: "Agradeciendo su presencia se les reitera la importancia y la finalidad del experimento en que ustedes son partícipes. Para nosotros es muy importante que ustedes estén cómodos y tengan lo necesario mientras esperan su turno". Posteriormente, los jóvenes fueron tratados con las mismas condiciones de la primera fase. Terminando la sesión se le agradeció su participación y se les proporcionó un cuestionario que contestaron de manera individual.

\section{Diseño experimental}

Los sujetos fueron expuestos a dos fases de experimentación. Cada fase tuvo una duración de 50 minutos. En cada fase experimental los sujetos estuvieron expuestos a un período de libre acceso de alimento y bebida. En la primera fase se proyectó un DVD de videos musicales, y en la segunda fase un DVD que contenía información sobre efectos negativos en el consumo excesivo de papas fritas y refresco de cola. Esta última fase se realizó una semana después de la exposición de los sujetos a la primera fase (Tabla 1).

\section{Análisis de la información}

Se analizaron los tres registros de grabación mediante el programa The Observer contabilizando la cantidad y preferencia de alimento y bebida de cada individuo. Se consideró como pieza consumida a la porción completa de papa frita o fruta. En caso de consumir porciones incompletas se contabilizó como $1 \frac{1}{2}$ pieza. En cuanto a la bebida, se contabilizó como una porción cada botella que fue destapada y consumida. Se realizó la comparación de los datos obtenidos entre la primera y segunda fase experimental, señalando las diferencias de consumo de alimentos y bebidas para valorar la influencia que proporcionó la información no especifica presentada durante la segunda fase. Se utilizó el programa Sigma Plot, para la elaboración de tablas y gráficas comparativas. 
Tabla 1. Descripción de las fases del diseño experimental

FASE 1

Libre acceso alimento y bebida y la transmisión DVD videos musicales.
FASE 2

Libre acceso alimento y bebida y la transmisión del DVD información efectos negativos en la ingesta de papas fritas y refresco de cola.

\begin{tabular}{|c|c|c|}
\hline Duración: & 50 minutos & 50 minutos \\
\hline
\end{tabular}

\section{Resultados}

La Figura 1 muestra el registro del consumo de piezas de fruta, la Figura 2 de las piezas de papas fritas, la Figura 3 muestra el consumo total de alimento por género. Las columnas de la izquierda muestran los datos de las mujeres y las columnas de la derecha los de los hombres. En las Figuras 1 y 2 las mujeres son representadas con círculos blancos y los hombres con rombos grises. En la Figura 3 las mujeres son representadas con una barra blanca y los hombres con una barra gris.

La Figura 1 muestra el registro de la cantidad de piezas de fruta consumida por mujeres y hombres durante las Fases 1 (panel superior) y 2 (panel inferior). En la Fase 1 se observa que el consumo inició en el minuto 12, dos minutos después de que el alimento estuvo disponible. En ese minuto el consumo registrado fue igual en ambos géneros. La mayor cantidad de piezas de fruta consumida en un minuto entre mujeres y hombres, fue de 7 piezas. Durante el minuto 16 se presentó nuevamente un aumento en la cantidad de piezas consumidas. Durante el minuto 36 se presentó el último consumo y fue realizado por los hombres.

En la Fase 2 se observa que el consumo inició durante el minuto 15, tres minutos después de haberse expuesto el alimento a los sujetos. La mayor cantidad de piezas consumidas en un minuto entre mujeres y hombres fue de 7 piezas. La cantidad de piezas de fruta consumida fue mayor del minuto 19 al 30 . En el minuto 38 se presentó el último consumo y fue hecho por una mujer.

La Figura 2 muestra el registro de la cantidad de piezas de papas fritas que fueron consumidas por mujeres y hombres durante la Fase 1 y 2 . En la Fase 1 las mujeres iniciaron el consumo durante el minuto 14 , cuatro minutos después de haberse presentado el alimento. Mientras que los hombres lo hicieron en el minuto 19. La mayor cantidad de piezas de papas fritas consumidas en un minuto entre mujeres y hombres fue de 8 piezas. Los hombres consumieron papas fritas en menores cantidades en comparación con las mujeres y con lapsos de tiempo mayores entre consumo y consumo. En esta fase un hombre y una mujer no consumieron. La cantidad de piezas de papas fritas consumidas fue mayor del minuto 17 al minuto 28. En el minuto 38 se efectuó el último consumo y fue emitido por las mujeres.

En la Fase 2, las mujeres iniciaron su consumo durante el minuto 13 , tres minutos después de haberse presentado el alimento. Mientras que los hombres lo hicieron en el minuto 15. La mayor cantidad de piezas consumidas en un minuto entre mujeres y hombres fue de 6 piezas. Las mujeres consumieron mayor cantidad por minuto, hasta 5 piezas. Mientras que los hombres consumieron por minuto un máximo de 3 piezas. Durante el minuto 39 se presentó el último consumo y lo hicieron por los hombres.

En la Figura 3 se muestra el total de consumo de piezas de frutas y papas fritas que fueron consumidas en la Fase 1 y 2 por ambos géneros. La cantidad total de piezas de fruta consumida en la primera fase fue de 65, de las cuales los hombres consumieron 36 piezas y las mujeres 29 piezas. En la segunda fase el total fue de 41 , de las cuales los hombres consumieron 8 piezas y las mujeres 33 piezas.

El total de piezas de papas fritas consumidas en la primera fase fue de 71 , de las cuales los hombres 
consumieron 18 piezas y las mujeres 53 . En la segunda fase fue de 55 , de las cuales los hombres consumieron 27 piezas y las mujeres 28.

La Tabla 2 muestra el registro de la porción de agua y refresco de cola que fue consumido por mujeres y hombres durante la Fase 1 y 2 . En la Fase 1 se observó que una mujer y un hombre tomaron 1 porción de agua cada uno. El total de porción de agua consumida en la primera fase fue de 2. En la Fase 2, dos hombres y dos mujeres tomaron cada uno 1 porción de agua. En esta fase el total de porciones de agua consumidas fue de 4 .

Respecto al consumo de refresco de cola, en la Fase 1 únicamente se presentó un consumo de 1 porción, efectuada por una mujer. En la Fase 2 nuevamente sólo se hizo un consumo de una porción de refresco de cola realizado por una mujer.
La Tabla 3 muestra las respuestas al cuestionario aplicado a los 5 sujetos de manera individual al finalizar ambas fases del experimento. En dicho cuestionario el total de los sujetos respondieron que sí desayunaron, variando los horarios entre 6:30 a.m. y 11:30 a.m. Respecto a lo que prefirieron para beber: cuatro sujetos señalaron que suelen tomar agua y sólo uno refirió tomar refresco. El total de los sujetos reportó que prefiere tomar agua cuando come fruta. El total de los sujetos refirió preferir tomar refresco cuando comen frituras, aunque dos sujetos además de refresco también tomaron agua. De los alimentos presentados en el experimento, un sujeto reportó no tener gusto por la naranja. Otro reportó que sólo toma refresco de cola, quien en ambas fases consumió refresco de cola, y un sujeto refirió no tener gusto por el refresco de cola. Tres sujetos desayunaron aproximadamente dos horas antes de asistir a ambas fases del experimento.

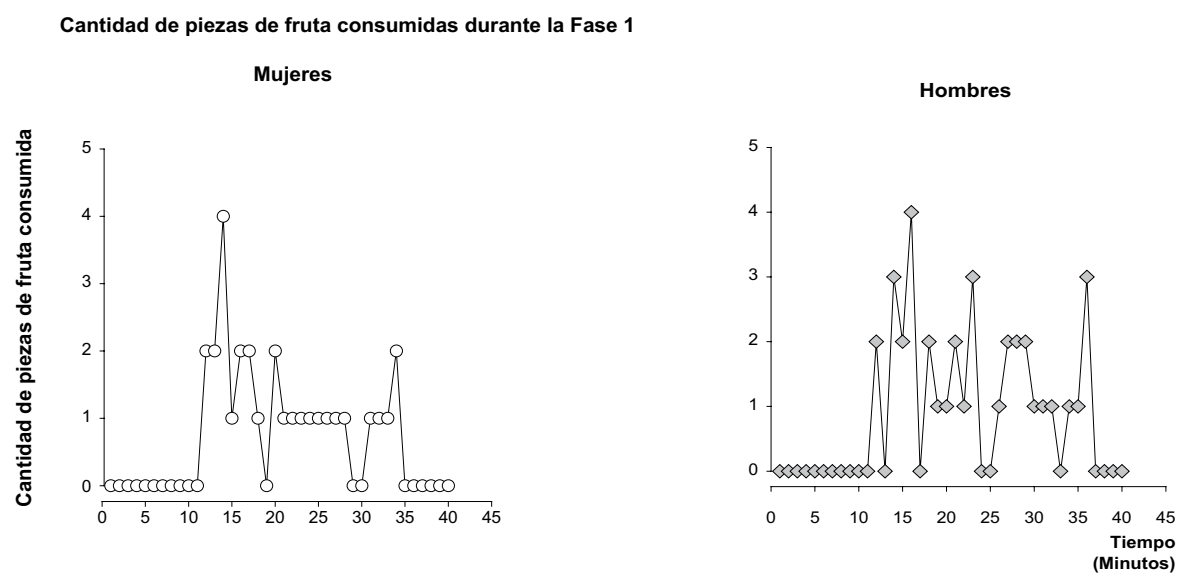

Cantidad de piezas de fruta consumidas durante la Fase 2
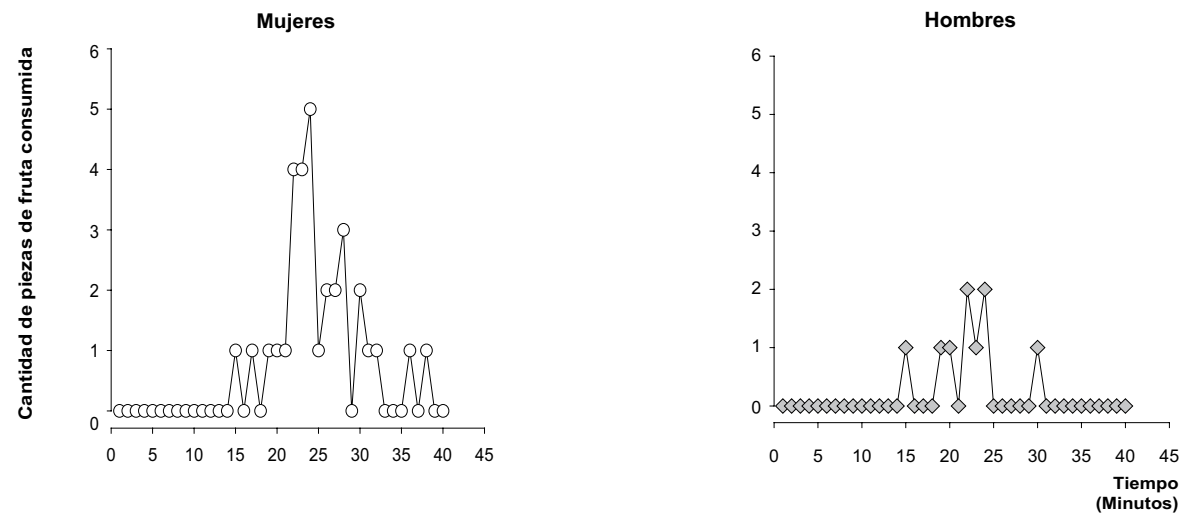

Figura 1. Cantidad de piezas de fruta consumidas por mujeres y hombres en la Fase 1 y 2 . La columna de la izquierda muestra los datos de las mujeres y la columna de la derecha los de los hombres. Las mujeres son representadas con círculos blancos y los hombres son representados con rombos grises. 
Cantidad de piezas de papas fritas consumidas durante la Fase 1
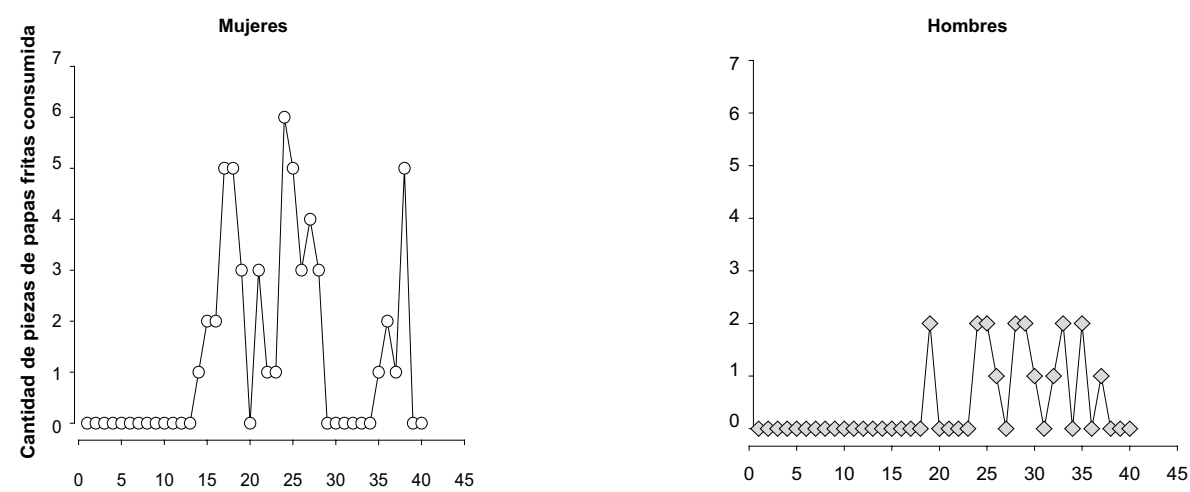

Cantidad de piezas de papas fritas consumidas durante la Fase 2
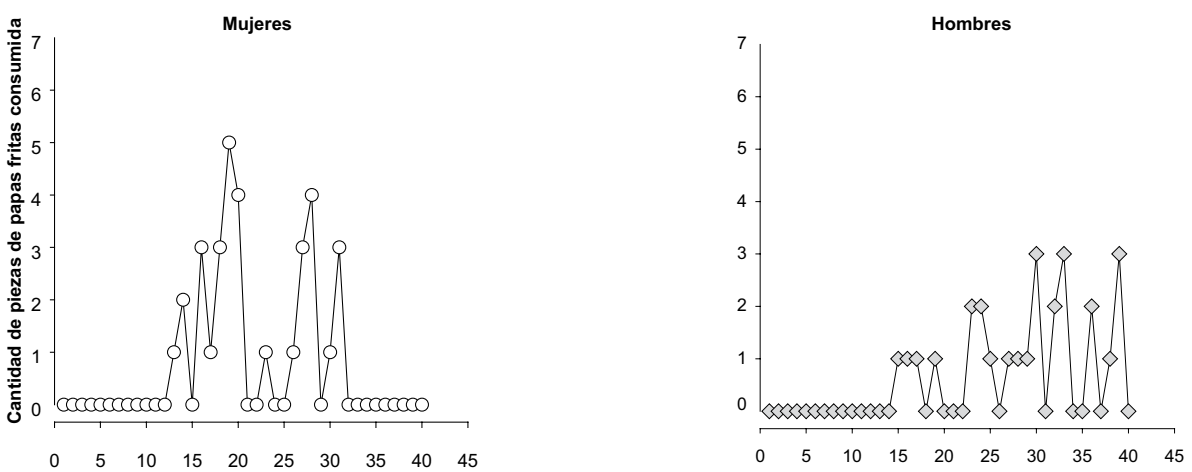

Figura 2. Cantidad de piezas de papas fritas consumidas por mujeres y hombres en la Fase 1 y 2 . La columna de la izquierda muestra los datos de las mujeres y la columna de la derecha los de los hombres. Las mujeres son representadas con círculos blancos y los hombres son representados con rombos grises.

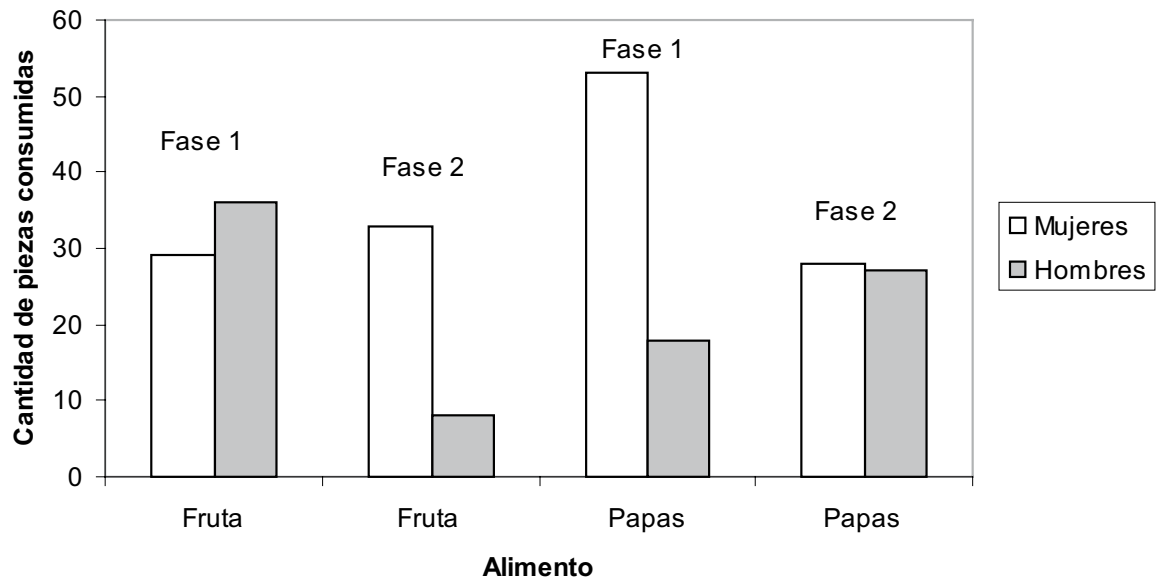

Figura 3. Total de alimento consumido por género en las Fases 1 y 2. 
Tabla 2. Porción de líquido consumido por mujeres y hombres en las Fases 1 y 2

\begin{tabular}{lcc}
\hline & Agua & Refresco de cola \\
\hline Fase 1 & 1 & 1 \\
Mujeres & 1 & 0 \\
Hombres & 1 & 1 \\
Total & 2 & \\
Fase 2 & & 1 \\
Mujeres & 2 & 0 \\
Hombres & 2 & 1 \\
\hline Total & 4 & \\
\hline
\end{tabular}

Tabla 3. Cuestionario aplicado a los sujetos experimentales

\begin{tabular}{|c|c|c|c|c|c|}
\hline PREGUNTAS & S1 & S2 & S3 & S4 & S5 \\
\hline Sexo & $\mathrm{H}$ & $\mathrm{H}$ & M & M & M \\
\hline Edad & 22 & 19 & 24 & 22 & 24 \\
\hline Estado civil & Soltero & Soltero & Soltera & Soltera & Soltera \\
\hline Ocupación & Estudiantes & Estudiante & Psicóloga & Estudiante & Psicóloga \\
\hline Grado académico & Universitario & Universitario & Licenciatura & Universitaria & Licenciatura \\
\hline ¿Sueles desayunar? & $\mathrm{Si}$ & $\mathrm{Si}$ & $\mathrm{Si}$ & $\mathrm{Si}$ & $\mathrm{Si}$ \\
\hline ¿A qué hora desayunas? & $6: 30 \mathrm{am}$ & $11: 30 \mathrm{am}$ & $9: 00 \mathrm{am}$ & $8: 00 \mathrm{am}$ & $8: 00 \mathrm{am}$ \\
\hline $\begin{array}{l}\text { ¿Qué desayunas } \\
\text { generalmente? }\end{array}$ & No contestó & Huevos, leche & $\begin{array}{l}\text { Cereal, } \\
\text { quesadillas, } \\
\text { yogurt }\end{array}$ & $\begin{array}{l}\text { Cereal, galletas, } \\
\text { quesadillas }\end{array}$ & Huevos, tacos \\
\hline $\begin{array}{l}\text { ¿Sueles comer entre } \\
\text { comidas? }\end{array}$ & En la tarde & Sí & Sí & Sí & Sí \\
\hline $\begin{array}{l}\text { ¿Qué tipo de frituras } \\
\text { prefieres? }\end{array}$ & Palomitas & Papas & Papas fritas & Papas fritas & Papas \\
\hline $\begin{array}{l}\text { ¿Qué día de la semana sueles } \\
\text { comer fruta? }\end{array}$ & Miércoles & Viernes & No especifico & Varia & Domingo \\
\hline $\begin{array}{l}\text { ¿A qué hora del día sueles } \\
\text { comer frituras? }\end{array}$ & $6: 00 \mathrm{pm}$ & $5: 00 \mathrm{pm}$ & No contestó & Tarde & 7:00 pm \\
\hline ¿Qué líquido sueles tomar? & Agua & Agua & Agua & Agua & Refresco \\
\hline $\begin{array}{l}\text { ¿Qué liquido prefieres tomar } \\
\text { por la mañana? }\end{array}$ & Leche & Agua, leche & Jugo, café & Agua & Refresco \\
\hline ¿Qué frutas te gustan más? & $\begin{array}{l}\text { Mango, pepino, } \\
\text { plátano }\end{array}$ & papaya, mango & $\begin{array}{l}\text { Manzana, fresa, } \\
\text { sandia, papaya }\end{array}$ & $\begin{array}{l}\text { Naranja, mango, } \\
\text { melón }\end{array}$ & Sandia, mango \\
\hline ¿Qué frutas no te gustan? & Naranja, papaya & Piña & $\begin{array}{l}\text { Kiwi, piña, } \\
\text { mamey }\end{array}$ & Ninguna & Durazno, fresa \\
\hline $\begin{array}{l}\text { ¿Qué líquido prefieres tomar } \\
\text { cuando comes fruta? }\end{array}$ & Agua & Agua & Agua & Agua & Agua \\
\hline $\begin{array}{l}\text { ¿Qué liquido prefieres tomar } \\
\text { cuando comes frituras? }\end{array}$ & Refresco & Refresco & Agua, refresco & Agua, refresco & Refresco \\
\hline $\begin{array}{l}\text { Del alimento que se te } \\
\text { ofreció en este experimento, } \\
\text { ¿Hubo alguno que no te } \\
\text { guste? }\end{array}$ & Sí & No & Sí & No & No \\
\hline Cuál? & Papas fritas & & Refresco de cola & & \\
\hline ¿Por qué? & $\begin{array}{l}\text { No las como por } \\
\text { la mañana }\end{array}$ & & $\begin{array}{l}\text { No me gusta } \\
\text { sabor, }\end{array}$ & & \\
\hline $\begin{array}{l}\text { ¿Consumiste algún alimento } \\
\text { o bebida la semana pasada } \\
\text { antes de dicho experimento? }\end{array}$ & Sí & No & No & Sí & Sí \\
\hline ¿Qué consumiste? & Cereal & & & Yogurt & Tacos \\
\hline ¿A qué hora? & $8: 00 \mathrm{am}$ & & & $8: 00 \mathrm{am}$ & $9: 00 \mathrm{am}$ \\
\hline $\begin{array}{l}\text { ¿Consumiste algún alimento } \\
\text { o bebida antes de dicho } \\
\text { experimento el día de hoy? }\end{array}$ & Sí & No & No & Sí & Sí \\
\hline ¿Qué consumiste? & Cereal & & & Cereal de avena & Tacos \\
\hline ¿A qué hora? & 8:00 am & & & $8: 00 \mathrm{am}$ & 9:00 am \\
\hline
\end{tabular}




\section{Discusión}

Los resultados obtenidos mostraron que en la Fase 1 la cantidad de piezas de fruta consumidas fue mayor durante los primeros 20 minutos del experimento, mientras que en la Fase 2 la mayor cantidad de piezas de fruta consumidas fue del minuto 24 al 30. En ambas fases la mayor cantidad de piezas de fruta consumidas entre mujeres y hombres durante un minuto fue de 7 piezas.

Un fenómeno observado fue la diferencia en la cantidad de piezas de fruta consumida de acuerdo al género. En la segunda fase las mujeres aumentaron la cantidad de piezas de fruta consumidas y los hombres disminuyeron la cantidad de piezas de fruta consumidas. Adicionalmente, la cantidad de piezas de fruta consumidas fue menor en la segunda fase en comparación con la primera fase.

Respecto al consumo de papas fritas también se observaron diferencias en la cantidad e intervalos de tiempo por género. Las mujeres consumieron más piezas de papas fritas en una menor cantidad de tiempo que los hombres. En comparación con la Fase 1, en la Fase 2 el consumo de papas disminuyó en las mujeres y aumentó en los hombres.

Estos datos sugieren que las instrucciones audiovisuales no explícitas tienen un efecto diferente en el consumo de alimento nutritivo y no nutritivo en hombres y mujeres. Al respecto Martínez (2001) señaló que una misma instrucción puede ejercer control sobre ejecuciones distintas en diferentes sujetos, así como también, que la ejecución de una misma instrucción puede variar de acuerdo con el momento en que sea emitida. No obstante, los resultados hacen referencia a las instrucciones no explícitas, los datos obtenidos en este trabajo parecen acordes con lo antes mencionado.

Store et al. (2002) distinguieron cuatro niveles de factores que influyen en la conducta alimentaria: 1) el individuo, 2) el ambiente social, 3) el ambiente físico, y, 4) el macro sistema. Esta clasificación podría explicar la influencia de la información recibida por los medios de comunicación y su impacto en la conducta alimentaria.
Silverio \& Alves (2004) señalaron que las instrucciones no específicas utilizadas en dietas para perder peso, anunciadas en publicaciones nocientíficas, influyen en la adopción de conductas alimentarias. Nuestros resultados reflejaron que también la información sobre los efectos en la salud por el consumo excesivo de comida con poco valor nutricional modifica el consumo alimenticio, al menos mientras se observó dicha información.

Taras et al. (1989) refieren que la mayor parte del tiempo, los medios de comunicación funcionan como instrucciones no específicas promoviendo conductas alimenticias. A partir de esto, cabe preguntarse si la conducta alimenticia promovida por las instrucciones audiovisuales no explicitas persiste a pesar de no ser reforzada. En nuestro estudio, la cantidad de piezas de papas fritas consumidas disminuyó mientras los participantes observaban el audiovisual. Por tanto, es necesario realizar más estudios en los que se evalúe si dicha conducta persiste y por cuánto tiempo a pesar de no ser reforzada.

Bellisle et al. (2000) señalaron que cuando el alimento es degustable, la gente come más y en un menor período de tiempo, ya que se mastica más rápido. Agregaron que se come una mayor cantidad en los primeros minutos de exposición a la comida. Encontraron que la textura es un factor crítico para la ingesta de alimento. Nuestros resultados sugieren que en un minuto se consume mayor cantidad de piezas de papas fritas que de piezas de fruta. Consideramos que esto se debe a la textura y la masa, esto es, que la composición de la fruta es más densa.

Young (1966) y Long et al. (2003) señalaron la importancia de informar a las personas sobre los aportes nutricios y sus beneficios en la dieta diaria, subrayando que la elección del alimento está directamente relacionada con el aprendizaje. En la Fase 2 de nuestro experimento, el video presentado hizo referencia a los efectos secundarios del consumo excesivo de comida con poco valor nutricional (papas fritas, bollos, refresco). Se mostraron estadísticas del valor nutricional de algunos alimentos empacados, así como registros médicos (rendimiento físico, cambios químicos, aumento 
de peso, estado emocional) de una persona que come únicamente alimentos con poco valor nutricional. En esta fase nuestros registros mostraron que la cantidad de alimento consumido fue menor, tanto de papas fritas como las piezas de fruta. No obstante, en el transcurso del experimento -cuando en el programa presentado hizo referencia a los efectos secundarios del consumo de alimentos con poco valor nutricional-, los sujetos consumieron mayor cantidad de piezas de fruta. Sin embargo, nuestros registros demostraron que en ambas fases fue mayor la cantidad de piezas de papas fritas consumidas que la cantidad de piezas de fruta consumidas, a pesar de que en la Fase 2 la cantidad de piezas de papas fritas consumidas disminuyó en mayor proporción que la cantidad de piezas de fruta consumidas. También se observó que cuando la cantidad de piezas de papas fritas consumidas aumentaba, la cantidad de piezas de fruta consumidas disminuía y viceversa. Sólo una mujer en la segunda fase consumió la misma cantidad de piezas de fruta y de papas fritas.

Al respecto, Martínez et al. (2002) y Ortiz, González \& Rosas (en Ortiz, 2005) señalaron la importancia de emitir una instrucción de manera específica. Además, afirmaron que mientras más específica sea una instrucción, los resultados serán más efectivos. También señalaron los elementos que debe tener una instrucción: presencia, relevancia, especificidad y pertinencia. $Y$ en base a estos propusieron seis categorías. Tomando en cuenta dichos elementos, la instrucción utilizada en nuestro experimento fue irrelevante ya que no tuvo relación con el objetivo de estudio. Szczurek (1978) y Gagne (1987) subrayaron la distinción entre instrucción como proceso e instrucción como producto, nos referiremos al segundo que hace referencia a un conocimiento claro y firme, al hecho de dar una indicación. En las instrucciones emitidas a lo largo de nuestro experimento se omitió el objetivo de este estudio ya que el uso de la instrucción depende del objetivo planteado (Martínez, 2001).

Un fenómeno de relevancia observado en ambas fases es el proceso de interacción social al introducir el alimento. Se observó que la presencia de alimento facilitó la interacción entre los sujetos, disminuyendo dicha interacción cuando el consumo de alimento aumentó. Sin embargo, los sujetos que presentaron mayor cantidad de interacciones también presentaron mayor cantidad de piezas de alimento consumido con predominio de papas fritas. También, se observó que la interacción fue mayor entre las mujeres. Fueron ellas quienes iniciaron con la interacción. Dicho proceso no fue objeto de estudio en este experimento. Se sugiere desarrollar posteriores estudios que contribuyan a evaluar el papel del alimento en el proceso de interacción.

Es necesario señalar que los participantes en este experimento pertenecen a un rango de edad de 19 a 24 años, de un nivel socioeconómico medio, todos solteros y estudiantes universitarios o profesionales. Cabero (1989) señaló que las cuatro dimensiones que interactúan entre sí y que determinan los productos a alcanzar en un proceso instruccional son: el alumno; video; uso concedido al video; $y$, contexto instruccional. Por otra parte, Edmonds et al. (1994) señalaron que se debe tener en cuenta los conocimientos previos y las habilidades asociadas de quienes participarán en el proceso de instrucción. Por su parte, Baron \& Galizio (1983) y Martínez \& Ribes (1996) mencionaron la importancia de considerar como variables la historia individual, la instrucción específica y la historia del reforzamiento del seguimiento instruccional a fin de obtener la conducta deseada al emitir una instrucción.

Al finalizar ambas fases del experimento se aplicó un cuestionario en el cual se solicitaron datos personales, preferencia de alimento y bebi$\mathrm{da}$, gusto o disgusto por los alimentos y bebidas presentados en el experimento $y$, finalmente, se preguntó si habían desayunado antes de asistir al experimento. En los resultados se observó que todos los individuos tienen hábitos alimenticios similares: no suelen comer entre comidas, mayor consumo de alimento nutritivo, prefieren tomar agua. Solo una persona no consumió una de las frutas presentadas en el experimento, y otra persona no consumía refresco de cola.

Finalmente, los resultados obtenidos en esta investigación indican que las instrucciones audio- 
visuales no explícitas modifican la conducta de consumo. Sin embargo, es necesario evaluar si estas conductas de consumo pueden persistir y por cuanto tiempo. Una propuesta es evaluar el comportamiento de una persona cuando deja de estar expuesta a las instrucciones audiovisuales con información explícita, como los efectos secundarios por el excesivo consumo de comida no saludable. Por tanto, es importante desarrollar otros estudios que contribuyan a la explicación de la permanencia de dicha conducta.

\section{Referencias}

Baron, A. \& Galizio, M. (1983). Instructional control of human operant behavior. Psychological Record, 33, 495-520.

Bellisle, F., Guy-Grand, B. \& Le Magnen, J. (2000). Chewing and swallowing as indices of the stimulation to eat during meals in humans: effects revealed by the edogram method and video recordings. Neurociencience and Biobehavioral Review, 24, 223-228.

Cabero, J. (1989). Tecnología Educativa: utilización didáctica del vídeo. Barcelona: PPU.

Edmonds G., Branch R. \& Mukherjee P. (1994). A Conceptual Framework for Comparing Instructional Design Models. Englewood Cliffs: Educational Technology Research y Development, 42, 4.

Gagné, R. (1987). Las condiciones del aprendizaje. México: Editorial Interamericana.

Long, C., Montano, A., Conklin, N. \& Scheer, S. (2003). The influence of healthy eating for life programs on eating behaviors of nonmetropolitan congregate meal participants. Family Economics and Nutrition Review, 15, 1, 15-24.

Martínez, H. (2001). Estudios sobre transferencia en comportamiento humano. En: Mares, G. \& Guevara, Y. (Eds). Psicología Interconductual, vol. I. México, UNAM.
Martínez, H., Ortiz, G. \& González, A. (2002). Precisión instruccional, retroalimentación y eficacia: efectos sobre el entrenamiento y transferencia en una tarea de discriminación condicional en adultos. Acta Colombiana de Psicología, 8, 7-33.

Martínez, H. \& Ribes, E. (1996). Interaction of contingencias and isntructional history on condicional discrimination. The Psychological Record, 46, 301-318.

Organización Mundial de la Salud (OMS) y Organización de las Naciones Unidas para la Agricultura y la Alimentación (FAO). (2005). Un marco para la promoción de frutas y verduras a nivel nacional. Organización Panamericana de la Salud (trad.), pp. 2-29.

Ortiz, G. A. (2005). El papel de la retroalimentación y la precisión instruccional en la elaboración y uso de descripciones en tareas de discriminación condicional. (Tesis doctoral inédita). Universidad de Guadalajara, Jalisco, México.

Silverio, O. \& Alves, D. (2004). Weight loss diets advertised in non-scientific publications. Cad. Saúde Pública, Río de Janeiro, 20 (5), 1219-1222.

Store, M., Neumark-Sztainer, D., \& French, S. (2002). Individual and environmental influences on adolescent eating behaviors. Journal of the American Dietetic Association, 102 (3), S40-S51.

Szczurek, M. (1978). Tecnología educativa y tecnología instruccional. Revista de Tecnología Educativa, 4 (3), 257-251.

Taras, H., Sallis, J., Patterson, T., Nader, P. \& Nelson, J. (1989). Television's influence on children's diet and physical activity. Journal Development Behavior Pediatric, 10 (4), 176-80.

Young, P.T. (1996). Hedonic organization and regulation of behavior. Psychological Review, 73 (1), 59-86. 
